\title{
Quantum wave equation of non-conservative system
}

\author{
Xiang-Yao $\mathrm{Wu}^{a}{ }^{1}$, Bai-Jun Zhang ${ }^{a}$, Hai-Bo $\mathrm{Li}^{a}$ \\ Xiao-Jing $\mathrm{Liu}^{a}$, Jing-Wu Li ${ }^{a}$ and Yi-Qing Guo ${ }^{b}$ \\ a. Institute of Physics, Jilin Normal University, Siping 136000, China \\ b. Institute of High Energy Physics, P. O. Box 918(3), Beijing 100049, China
}

\begin{abstract}
It is well known that Schrödinger's equation is only suitable for the particle in conservative force field. In atomic and molecular field, a particle can suffer the action of non-conservative force. In this paper, a new quantum wave equation is proposed, which can describe the particle in non-conservative force field. We think the new quantum wave equation can be used in many fields.
\end{abstract}

PACS numbers: 03.65.Ta; 03.65.-w

Keywords: Non-Conservative System; Quantum Wave Equation

\footnotetext{
${ }^{1}$ E-mail:wuxy2066@163.com
} 


\section{Introduction}

It is well known that quantum mechanics (QM) acquired its final formulation in 19251926 through fundamental papers of Schrödinger and Heisenberg. Originally these papers appeared as two independent views of the structure of quantum mechanics, but in 1927 Schrödinger established their equivalence, and since then one or the other of the papers mentioned have been used to analyze quantum mechanical systems, depending on which method gave the most convenient way of solving the problem. In the 1940's Richard Feynman, and later many others, derived a propagator for quantum mechanical problems through a path integration procedure[1-3]. In contrast with the Hamiltonian emphasis in the original formulation of quantum mechanics, Feynman's approach could be referred to as Lagrangian and it emphasized the propagator $K\left(x, t ; x^{\prime}, t^{\prime}\right)$ which takes the wave function $\psi\left(x^{\prime}, t^{\prime}\right)$ at the point $x^{\prime}$ and time $t^{\prime}$ to the point $x$ at time $t$. While this propagator could be derived by the standard methods of quantum mechanics. Feynman invented a procedure by summing all time dependent paths connecting points $x, x^{\prime}$ and this became an alternative formulation of quantum mechanics whose results coincided with the older version when all of them where applicable.

Quantum Mechanics has become one of the most important foundations of physics, and achieved great success, physicists had begun to consider the possibility to generalize the traditional framework of it[4]. Up to now, this kind of attempts have never stopped[5-8]. As we known, Schrödinger's equation is only suitable for the particle lying in conservative force field. For a non-conservative system, a number of other methods have been proposed to study it. In Ref. [9], Riewe extend Lagrangian and Hamiltonian mechanics to include derivatives of fractional order. The fractional derivatives lead directly to equations of motion with nonconservative classical forces such as friction. In Ref. [10], Riewe continues the development of fractional-derivative mechanics by deriving a modified Hamiltons principle. By using fractional derivatives, it is possible to construct a complete mechanical description of nonconservative systems, including Lagrangian and Hamiltonian mechanics, canonical transformations, Hamilton-Jacobi theory, and quantum wave mechanics. The method is illustrated with a frictional force proportional to velocity. Besides the use of fractional derivatives, Bateman [11] suggested two methods based on the idea that a Lagrangian could lead to multiple equations. In Refs. [12, 13], Schrödinger's equation was modified directly. For example, a nonlinear term proportional to $\ln \left(\psi / \psi^{*}\right)$ can be added, and these types of modifications provide quantum results corresponding to classical friction. A standard device for dealing with dissipation is the Rayleigh dissipation function [14], which can be used when frictional forces are proportional to velocity.

In this paper, a new quantum wave equation is proposed by application of the Feynman's path-integral. The wave equation can describe the particle suffering the nonconservative force which is proportional to velocity. 


\section{The Lagrange function for conservative and non- conservative system}

The general Lagrange's equations are

$$
\frac{d}{d t} \frac{\partial T}{\partial \dot{q}_{j}}-\frac{\partial T}{\partial q_{j}}=Q_{j} \quad(j=1,2, \cdots, s),
$$

where the kinetic energy $T$ and the generalized forces $Q_{j}$ are, in general functions of all the generalized coordinates $q_{j}$ and generalized velocities $\dot{q}_{j}$ and of $t$. The $Q_{j}$ is defined as

$$
Q_{j}=\sum_{i=1}^{N} \vec{F}_{i} \cdot \frac{\partial \overrightarrow{r_{i}}}{\partial q_{j}}
$$

where $\vec{r}_{i}=\vec{r}_{i}\left(q_{1}, q_{2}, \cdots q_{s}, t\right)(i=1,2, \cdots N)$ and $\vec{F}_{i}$ is the external force acting on a particle. If all the $\vec{F}_{i}$ are conservative force, and then $\vec{F}_{i}=-\nabla_{i} V$, we can write

$$
Q_{j}=\sum_{i} \vec{F}_{i} \cdot \frac{\partial \overrightarrow{r_{i}}}{\partial q_{j}}=-\sum_{i} \nabla_{i} V \cdot \frac{\partial \overrightarrow{r_{i}}}{\partial q_{j}}=-\frac{\partial V}{\partial q_{j}},
$$

where the $V$ is a scalar potential function, and so Eq. (1) becomes

$$
\frac{d}{d t} \frac{\partial T}{\partial \dot{q}_{j}}-\frac{\partial T}{\partial q_{j}}+\frac{\partial V}{\partial q_{j}}=0
$$

since $V$ is a function of the $\vec{r}_{i}$ and therefore only of the $q_{j}$ and not the $\dot{q}_{j}$, we have $\frac{\partial V}{\partial \dot{q}_{j}}=0$. Let us now define the Lagrangian function $L$ and action $S$ of the system as

$$
L=T-V
$$

and

$$
S=\int_{t_{1}}^{t_{2}} L d t
$$

and then in terms of this function the equations of motion become

$$
\frac{d}{d t} \frac{\partial L}{\partial \dot{q}_{j}}-\frac{\partial L}{\partial q_{j}}=0 \quad(j=1,2, \cdots, s) .
$$

These are called Lagrange's equations. If the $\vec{F}_{i}(i=1,2, \cdots N)$ include conservative force and non-conservative force, we can have

$$
Q_{j}=Q_{j}^{(1)}+Q_{j}^{(2)},
$$

and

$$
Q_{j}^{(1)}=-\frac{\partial V}{\partial q_{j}}
$$


where the $Q_{j}^{(1)}$ is conservative generalized force, and $Q_{j}^{(2)}$ is non-conservative generalized force. The Lagrange's equations become

$$
\frac{d}{d t} \frac{\partial L}{\partial \dot{q}_{j}}-\frac{\partial L}{\partial q_{j}}=Q_{j}^{(2)} \quad(j=1,2, \cdots, s) .
$$

For a conservative system, we can define the Lagrangian function $L$ and action $\mathrm{S}$ as Eq.(5) and (6). For a generalized system, i.e.,including conservative and non-conservative force, we can define the general Lagrangian function $L$ as:

$$
L=T+\int_{P_{0}}^{A} \vec{F}_{c} \cdot d \vec{r}-\int_{P_{0}}^{A} \vec{F}_{n c} \cdot d \vec{r}
$$

where the $\vec{F}_{c}$ is conservative force and the $\vec{F}_{n c}$ is non-conservative force, the point $P_{0}$ is the reference point, and the $A$ is the position point for a particle in space. If the system is conservative, the Eq. (11) becomes

$$
\begin{aligned}
L & =T+\int_{P_{0}}^{A} \vec{F} \cdot d \vec{r} \\
& =T-V_{A}
\end{aligned}
$$

where $V_{A}$ is potential energy of the system. In this case, the Eq. (11) becomes Eq. (5), and then Eq. (11) can be written as:

$$
L=T-V-\int_{P_{0}}^{A} \vec{F}_{n c} \cdot d \vec{r} .
$$

\section{The quantum wave equation for non-conservative system}

In the following, we will deduce the quantum wave equation for the system that there are both conservative and non-conservative force with the approach of path integral. The path-integral formula is

$$
\Psi\left(\vec{r}, t^{\prime}\right)=\iint \exp \left[\frac{i}{\hbar} \int_{t}^{t^{\prime}} L(\dot{\vec{r}}(\tau), \vec{r}(\tau), \tau) d \tau\right] D[\vec{r}(t)] \Psi\left(\vec{r}^{\prime}, t\right) d \vec{r}^{\prime},
$$

which gives the wave function at a time $t^{\prime}$ in terms of the wave function at a time $t$. In order to obtain the differential equation, we apply this relationship in the special case that the time $t^{\prime}$ differs only by an infinitesimal interval $\varepsilon$ from $t$. For a short interval $\varepsilon$ the action is approximately $\varepsilon$ times the Lagrangian for this interval, we have

$$
\Psi(\vec{r}, t+\varepsilon)=\int \frac{d \vec{r}^{\prime}}{A^{3}} \exp \left[\frac{i \varepsilon}{\hbar} L\left(\frac{\vec{r}-\vec{r}^{\prime}}{\varepsilon}, \frac{\vec{r}+\vec{r}^{\prime}}{2}, \frac{t^{\prime}+t}{2}\right)\right] \Psi\left(\vec{r}^{\prime}, t\right),
$$

where $A$ is a normalization constant. Substituting Eq. (13) into (15), one can obtain

$$
\Psi(\vec{r}, t+\varepsilon)=\int \frac{d \vec{r}^{\prime}}{A^{3}} \exp \left[\frac{i \varepsilon}{\hbar}\left(\frac{m}{2}\left(\frac{\vec{r}-\vec{r}^{\prime}}{\varepsilon}\right)^{2}-V\left(\frac{\vec{r}+\vec{r}^{\prime}}{2}, \frac{t^{\prime}+t}{2}\right)-\int_{\vec{r}^{\prime}}^{\vec{r}} \vec{F} \cdot d \vec{r}^{\prime \prime}\right)\right] \Psi\left(\vec{r}^{\prime}, t\right) .
$$


In macroscopic field, the frictional force and adhere force are non-conservative force, and the non-conservative force $\vec{F}$ is directly proportional to velocity $\vec{v}$, their directions are opposite, i.e., $\vec{F}=-k \vec{v}$. In microscopic field, a particle can also suffer the action of non-conservative force. The nonconservative quantum processes are common too, since there is dissipation in every nonequilibrium or fluctuating process, including tunnelling [15], electromagnetic cavity radiation [16, 17], masers and parametric amplification [17], Brownian motion [12, 18], inelastic scattering [13, 19], squeezed states of quantum optics [20], and electrical resistance or Ohmic friction [21]. In the experiment of Bose-Einstein condensates, the atomic $R b^{87}, N a^{23}$ and $L i^{7}$ can be cooled in laser field, since they get the non-conservative force from the photons, and the force $\vec{F}=-k \vec{v}$. Substituting $\vec{F}=-k \vec{v}$ into Eq. (16), we have

$$
\begin{aligned}
\Psi(\vec{r}, t+\varepsilon)= & \int \frac{d \vec{r}^{\prime}}{A^{3}} \exp \left[\frac { i \varepsilon } { \hbar } \left(\frac{m}{2}\left(\frac{\vec{r}-\vec{r}^{\prime}}{\varepsilon}\right)^{2}-V\left(\frac{\vec{r}+\vec{r}^{\prime}}{2}, \frac{t^{\prime}+t}{2}\right)\right.\right. \\
& \left.\left.+k \int_{\vec{r}^{\prime}}\left(\frac{\vec{r}-\vec{r}^{\prime}}{\varepsilon}\right) \cdot d \vec{r}^{\prime \prime}\right)\right] \Psi\left(\vec{r}^{\prime}, t\right) \\
= & \int \frac{d \vec{r}^{\prime}}{A^{3}} \exp \left[\frac{i}{\hbar} \frac{m}{2} \frac{\left(\vec{r}-\vec{r}^{\prime}\right)^{2}}{\varepsilon}\right] \cdot \exp \left[-\frac{i \varepsilon}{\hbar} V\left(\frac{\vec{r}+\vec{r}^{\prime}}{2}, \frac{t+t^{\prime}}{2}\right)\right] \\
& \cdot \exp \left[\frac{i \varepsilon}{\hbar} k \int_{\vec{r}^{\prime}}^{\vec{r}} \frac{\vec{r}-\vec{r}^{\prime}}{\varepsilon} \cdot d \vec{r}^{\prime \prime}\right] \cdot \psi\left(\vec{r}^{\prime}, t\right) .
\end{aligned}
$$

The quantity $\frac{\left(\vec{r}-\vec{r}^{\prime}\right)^{2}}{\varepsilon}$ appears in the exponent of the first factor. It is clear that if $\vec{r}^{\prime}$ is appreciably different from $\vec{r}$, this quantity is very large and the exponential consequently oscillates very rapidly as $\vec{r}^{\prime}$ varies, when this factor oscillates rapidly, the integral over $\vec{r}^{\prime}$ gives a very small value. Only if $\vec{r}^{\prime}$ is near $\vec{r}$ do we get important contributions. For this reason we make the substitution $\vec{r}^{\prime}=\vec{r}+\vec{\eta}$ with the expectation that appreciable contribution to the integral will occur only for small $\vec{\eta}$, we obtain

$$
\begin{aligned}
\Psi(\vec{r}, t+\varepsilon)= & \int \frac{d \vec{\eta}}{A^{3}} \exp \left[\frac{i}{\hbar} \frac{m}{2} \frac{\vec{\eta}^{2}}{\varepsilon}\right] \cdot \exp \left[-\frac{i \varepsilon}{\hbar} V\left(\vec{r}+\frac{\vec{\eta}}{2}, t+\frac{\varepsilon}{2}\right)\right] \\
& \left.\cdot \exp \left[\frac{i \varepsilon}{\hbar} k \int_{\vec{r}^{\prime}}^{\vec{r}} \frac{-\vec{\eta}}{\varepsilon} \cdot d \vec{r}^{\prime \prime}\right)\right] \Psi(\vec{r}+\vec{\eta}, t)
\end{aligned}
$$

Now we have

$$
\int_{\vec{r}^{\prime}}^{\vec{r}} \vec{\eta} \cdot d \vec{r}^{\prime \prime}=\int_{\vec{r}^{\prime}}^{\vec{r}}|\vec{\eta}|\left|d \vec{r}^{\prime \prime}\right| \cos \theta=|\vec{\eta}| \int_{\vec{r}^{\prime}}^{\vec{r}}\left|d \vec{r}^{\prime \prime}\right| \cos \theta=-|\vec{\eta}|^{2}
$$

so that

$$
k \int_{\vec{r}^{\prime}}^{\vec{r}} \frac{-\vec{\eta}}{\varepsilon} \cdot d \vec{r}^{\prime \prime}=\frac{k}{\varepsilon}|\vec{\eta}|^{2}=\frac{k}{\varepsilon} \vec{\eta}^{2}
$$

substituting Eq. (20) into (18), we have

$$
\Psi(\vec{r}, t+\varepsilon)=\int \frac{d \vec{\eta}}{A^{3}} e^{\frac{i m \vec{\eta}^{2}}{2 \hbar \varepsilon}} e^{-\frac{i \varepsilon}{\hbar} V\left(\vec{r}+\frac{\vec{\eta}}{2}, t+\frac{\varepsilon}{2}\right)} e^{\frac{i}{\hbar} k \vec{\eta}^{2}} \Psi(\vec{r}+\vec{\eta}, t)
$$


The phase of the first exponential changes by the order of 1 radian when $|\vec{\eta}|$ is of the order $\sqrt{\frac{2 \hbar \varepsilon}{m}}$, so that most of the integral is contributed by values of $|\vec{\eta}|$ in this order. We may expand $\Psi$ in a power series, we need only keep terms of order $\varepsilon$. This implies keeping second-order terms in $\eta_{x}, \eta_{y}$ and $\eta_{z}$. Expanding the left-hand side to first order in $\varepsilon$ and the right-hand side to first order in $\varepsilon$ and second order in $\eta_{x}, \eta_{y}$ and $\eta_{z}$, we have

$$
\begin{gathered}
e^{-\frac{i \varepsilon}{\hbar} V\left(\vec{r}+\frac{\vec{\eta}}{2}, t+\frac{\varepsilon}{2}\right)}=1-\frac{i \varepsilon}{\hbar} V(\vec{r}, t) \\
e^{\frac{i}{\hbar} k \vec{\eta}^{2}}=1+\frac{i}{\hbar} k \vec{\eta}^{2} \\
\Psi(\vec{r}+\vec{\eta}, t)=\Psi(\vec{r}, t)+\vec{\eta} \cdot \frac{\partial \Psi(\vec{r}, t)}{\partial \vec{r}}+\frac{1}{2}\left(\eta_{x}^{2} \frac{\partial^{2} \Psi}{\partial x^{2}}+\eta_{y}^{2} \frac{\partial^{2} \Psi}{\partial y^{2}}+\eta_{z}^{2} \frac{\partial^{2} \Psi}{\partial z^{2}}\right)
\end{gathered}
$$

and

$$
\begin{aligned}
\Psi(\vec{r}, t)+\varepsilon \frac{\partial \Psi(\vec{r}, t)}{\partial t}= & \int \frac{d \vec{\eta}}{A^{3}} e^{\frac{i m \vec{\eta}^{2}}{2 \hbar \varepsilon}}\left(1-\frac{i \varepsilon}{\hbar} V(\vec{r}, t)\right)\left(1+\frac{i}{\hbar} k \vec{\eta}^{2}\right)\left[\Psi(\vec{r}, t)+\vec{\eta} \cdot \frac{\partial \Psi(\vec{r}, t)}{\partial \vec{r}}+\right. \\
& \left.\frac{1}{2}\left(\eta_{x}^{2} \frac{\partial^{2} \Psi}{\partial x^{2}}+\eta_{y}^{2} \frac{\partial^{2} \Psi}{\partial y^{2}}+\eta_{z}^{2} \frac{\partial^{2} \Psi}{\partial z^{2}}\right)\right] \\
= & \int \frac{d \vec{\eta}}{A^{3}} e^{\frac{i m \vec{\eta}^{2}}{2 \hbar \varepsilon}}\left(1+\frac{i}{\hbar} k \vec{\eta}^{2}-\frac{i \varepsilon}{\hbar} V(\vec{r}, t)+\frac{\varepsilon}{\hbar^{2}} k V(\vec{r}, t) \vec{\eta}^{2}\right) \\
& {\left[\Psi(\vec{r}, t)+\vec{\eta} \cdot \nabla \Psi(\vec{r}, t)+\frac{1}{2}\left(\eta_{x}^{2} \frac{\partial^{2} \Psi}{\partial x^{2}}+\eta_{y}^{2} \frac{\partial^{2} \Psi}{\partial y^{2}}+\eta_{z}^{2} \frac{\partial^{2} \Psi}{\partial z^{2}}\right)\right] }
\end{aligned}
$$

In order to evaluate the right-hand side of Eq. (25), we shall have to use four integrals

$$
\begin{gathered}
\int_{-\infty}^{\infty} d \eta_{x} e^{\frac{i m \eta_{x}^{2}}{2 \hbar \varepsilon}} \equiv A=\left(\frac{i 2 \pi \hbar \varepsilon}{m}\right)^{\frac{1}{2}}, \\
\int_{-\infty}^{\infty} d \eta_{x} \eta_{x} e^{\frac{i m \eta_{x}^{2}}{2 \hbar \varepsilon}}=0, \\
\int_{-\infty}^{\infty} d \vec{\eta} e^{\frac{i m \vec{\eta}^{2}}{2 \hbar \varepsilon}}=A^{3}=\left(\frac{i 2 \pi \hbar \varepsilon}{m}\right)^{\frac{3}{2}},
\end{gathered}
$$

and

$$
\int_{-\infty}^{\infty} d \eta_{x} \eta_{x}^{2} e^{\frac{i m \eta_{x}^{2}}{2 \hbar \varepsilon}}=\frac{i \hbar \varepsilon}{m}\left(\frac{i 2 \pi \hbar \varepsilon}{m}\right)^{\frac{1}{2}}
$$

In Eq. (25), we can easily find that the integrals of the terms $\frac{\varepsilon}{\hbar^{2}} k V(\vec{r}, t) \vec{\eta}^{2}$ and $\vec{\eta} \cdot \nabla \psi(\vec{r}, t)$ are either zero or $O\left(\varepsilon^{2}\right)$ from Eqs. (26)-(29), and they can be neglected in Eq. (25). The Eq. (25) becomes

$$
\begin{aligned}
\Psi(\vec{r}, t)+\varepsilon \frac{\partial \Psi(\vec{r}, t)}{\partial t}= & \int \frac{d \vec{\eta}}{A^{3}} e^{\frac{i m \vec{\eta}^{2}}{2 \hbar \varepsilon}}\left[1+\frac{i}{\hbar} k \vec{\eta}^{2}-\frac{i \varepsilon}{\hbar} V(\vec{r}, t)\right][\Psi(\vec{r}, t) \\
& \left.+\frac{1}{2}\left(\eta_{x}^{2} \frac{\partial^{2} \Psi}{\partial x^{2}}+\eta_{y}^{2} \frac{\partial^{2} \Psi}{\partial y^{2}}+\eta_{z}^{2} \frac{\partial^{2} \Psi}{\partial z^{2}}\right)\right]
\end{aligned}
$$




$$
\begin{aligned}
= & \int \frac{d \vec{\eta}}{A^{3}} e^{\frac{i m \vec{\eta}^{2}}{2 \hbar \varepsilon}}\left(1-\frac{i \varepsilon}{\hbar} V(\vec{r}, t)\right)(\Psi(\vec{r}, t) \\
& \left.+\frac{1}{2}\left(\eta_{x}^{2} \frac{\partial^{2} \Psi}{\partial x^{2}}+\eta_{y}^{2} \frac{\partial^{2} \Psi}{\partial y^{2}}+\eta_{z}^{2} \frac{\partial^{2} \Psi}{\partial z^{2}}\right)\right) \\
& +\frac{i}{\hbar} k \int \frac{d \vec{\eta}}{A^{3}} e^{\frac{i m \eta^{2}}{2 \hbar \varepsilon}} \vec{\eta}^{2}[\Psi(\vec{r}, t) \\
& \left.+\frac{1}{2}\left(\eta_{x}^{2} \frac{\partial^{2} \Psi}{\partial x^{2}}+\eta_{y}^{2} \frac{\partial^{2} \Psi}{\partial y^{2}}+\eta_{z}^{2} \frac{\partial^{2} \Psi}{\partial z^{2}}\right)\right]
\end{aligned}
$$

In Eq. (30), the first term is

$$
\begin{aligned}
& \int \frac{d \vec{\eta}}{A^{3}} e^{\frac{i m \vec{\eta}^{2}}{2 \hbar \varepsilon}}\left(1-\frac{i \varepsilon}{\hbar} V(\vec{r}, t)\right) \Psi(\vec{r}, t) \\
= & \left(1-\frac{i \varepsilon}{\hbar} V(\vec{r}, t)\right) \Psi(\vec{r}, t) \int \frac{d \vec{\eta}}{A^{3}} e^{\frac{i m \vec{\eta}^{2}}{2 \hbar \varepsilon}} \\
= & \left(1-\frac{i \varepsilon}{\hbar} V(\vec{r}, t)\right) \Psi(\vec{r}, t),
\end{aligned}
$$

and the second term is

$$
\begin{aligned}
& \frac{1}{2} \int \frac{d \vec{\eta}}{A^{3}} e^{\frac{i m \vec{\eta}^{2}}{2 \hbar \varepsilon}}\left(1-\frac{i \varepsilon}{\hbar} V(\vec{r}, t)\right)\left(\eta_{x}^{2} \frac{\partial^{2} \Psi}{\partial x^{2}}+\eta_{y}^{2} \frac{\partial^{2} \Psi}{\partial y^{2}}+\eta_{z}^{2} \frac{\partial^{2} \Psi}{\partial z^{2}}\right) \\
= & \frac{1}{2} \int \frac{d \vec{\eta}}{A^{3}} e^{\frac{i m \vec{\eta}^{2}}{2 \hbar \varepsilon}} \eta_{x}^{2} \frac{\partial^{2} \Psi}{\partial x^{2}}+\frac{1}{2} \int \frac{d \vec{\eta}}{A^{3}} e^{\frac{i m \vec{\eta}^{2}}{2 \hbar \varepsilon}} \eta_{y}^{2} \frac{\partial^{2} \Psi}{\partial y^{2}}+\frac{1}{2} \int \frac{d \vec{\eta}}{A^{3}} e^{\frac{i m \vec{\eta}^{2}}{2 \hbar \varepsilon}} \eta_{z}^{2} \frac{\partial^{2} \Psi}{\partial z^{2}} .
\end{aligned}
$$

In Eq. (32), we can easily find that the integral of the term $\frac{i \varepsilon}{\hbar} V(\vec{r}, t)$ is $O\left(\varepsilon^{2}\right)$, which can be neglected. In Eq. (32), the first term is

$$
\frac{1}{2} \int \frac{d \vec{\eta}}{A^{3}} e^{\frac{i m \vec{\eta}^{2}}{2 \hbar \varepsilon}} \eta_{x}^{2} \frac{\partial^{2} \Psi}{\partial x^{2}}=\frac{1}{2} \frac{\partial^{2} \Psi}{\partial x^{2}} \int \frac{d \eta_{x}}{A} \eta_{x}^{2} e^{\frac{i m \eta_{x}^{2}}{2 \hbar \varepsilon}} \int \frac{d \eta_{y}}{A} e^{\frac{i m \eta_{y}^{2}}{2 \hbar \varepsilon}} \int \frac{d \eta_{z}}{A} e^{\frac{i m \eta_{z}^{2}}{2 \hbar \varepsilon}}=\frac{1}{2} \frac{\partial^{2} \Psi}{\partial x^{2}} \frac{i \hbar \varepsilon}{m}
$$

and similarly, the second and third terms are

$$
\begin{aligned}
& \frac{1}{2} \int \frac{d \vec{\eta}}{A^{3}} e^{\frac{i m \vec{\eta}^{2}}{2 \hbar \varepsilon}} \eta_{y}^{2} \frac{\partial^{2} \Psi}{\partial y^{2}}=\frac{1}{2} \frac{\partial^{2} \Psi}{\partial y^{2}} \frac{i \hbar \varepsilon}{m} \\
& \frac{1}{2} \int \frac{d \vec{\eta}}{A^{3}} e^{\frac{i m \vec{\eta}^{2}}{2 \hbar \varepsilon}} \eta_{z}^{2} \frac{\partial^{2} \Psi}{\partial z^{2}}=\frac{1}{2} \frac{\partial^{2} \Psi}{\partial z^{2}} \frac{i \hbar \varepsilon}{m}
\end{aligned}
$$

and so Eq. (32) becomes

$$
\frac{1}{2} \int \frac{d \vec{\eta}}{A^{3}} e^{\frac{i m \vec{\eta}^{2}}{2 \hbar \varepsilon}}\left(\eta_{x}^{2} \frac{\partial^{2} \Psi}{\partial x^{2}}+\eta_{y}^{2} \frac{\partial^{2} \Psi}{\partial y^{2}}+\eta_{z}^{2} \frac{\partial^{2} \Psi}{\partial z^{2}}\right)=\frac{1}{2} \frac{i \hbar \varepsilon}{m}\left(\frac{\partial^{2} \Psi}{\partial x^{2}}+\frac{\partial^{2} \Psi}{\partial y^{2}}+\frac{\partial^{2} \Psi}{\partial z^{2}}\right)=\frac{i \hbar}{2 m} \varepsilon \nabla^{2} \Psi
$$

In Eq. (30), the third term is

$$
\begin{aligned}
& \frac{i}{\hbar} k \int \frac{d \vec{\eta}}{A^{3}} e^{\frac{i m \vec{\eta}^{2}}{2 \hbar \varepsilon}} \vec{\eta}^{2} \Psi(\vec{r}, t) \\
= & \frac{i}{\hbar} k \Psi(\vec{r}, t)\left[\int \frac{d \vec{\eta}}{A^{3}} e^{\frac{i m \vec{\eta}^{2}}{2 \hbar \varepsilon}} \eta_{x}^{2}+\int \frac{d \vec{\eta}}{A^{3}} e^{\frac{i m \vec{\eta}^{2}}{2 \hbar \varepsilon}} \eta_{y}^{2}+\int \frac{d \vec{\eta}}{A^{3}} e^{\frac{i m \vec{\eta}^{2}}{2 \hbar \varepsilon}} \eta_{z}^{2}\right] .
\end{aligned}
$$


In Eq. (36), the first term is

$$
\int \frac{d \vec{\eta}}{A^{3}} e^{\frac{i m \vec{\eta}^{2}}{2 \hbar \varepsilon}} \eta_{x}^{2}=\int \frac{d \eta_{x}}{A} \eta_{x}^{2} e^{\frac{i m \eta_{x}^{2}}{2 \hbar \varepsilon}} \int \frac{d \eta_{y}}{A} e^{\frac{i m \eta_{y}^{2}}{2 \hbar \varepsilon}} \int \frac{d \eta_{z}}{A} e^{\frac{i m \eta_{z}^{2}}{2 \hbar \varepsilon}}=\frac{i \hbar \varepsilon}{m},
$$

and similarly, the second and third terms are

$$
\begin{aligned}
& \int \frac{d \vec{\eta}}{A^{3}} e^{\frac{i m \vec{\eta}^{2}}{2 \hbar \varepsilon}} \eta_{y}^{2}=\frac{i \hbar \varepsilon}{m} \\
& \int \frac{d \vec{\eta}}{A^{3}} e^{\frac{i m \vec{\eta}^{2}}{2 \hbar \varepsilon}} \eta_{z}^{2}=\frac{i \hbar \varepsilon}{m}
\end{aligned}
$$

and so Eq. (36) becomes

$$
\frac{i}{\hbar} k \int \frac{d \vec{\eta}}{A^{3}} e^{\frac{i m \vec{\eta}^{2}}{2 \hbar \varepsilon}} \vec{\eta}^{2} \Psi(\vec{r}, t)=\frac{i}{\hbar} k \Psi(\vec{r}, t) \frac{3 i \hbar \varepsilon}{m}=-\frac{3 \varepsilon k}{m} \Psi(\vec{r}, t) .
$$

In Eq. (30), the fourth term is

$$
\begin{aligned}
& \frac{i}{\hbar} k \int \frac{d \vec{\eta}}{A^{3}} e^{\frac{i m \vec{\eta}^{2}}{2 \hbar \varepsilon}} \vec{\eta}^{2} \frac{1}{2}\left(\eta_{x}^{2} \frac{\partial^{2} \Psi}{\partial x^{2}}+\eta_{y}^{2} \frac{\partial^{2} \Psi}{\partial y^{2}}+\eta_{z}^{2} \frac{\partial^{2} \Psi}{\partial z^{2}}\right) \\
= & \frac{i}{2 \hbar} k \int \frac{d \vec{\eta}}{A^{3}} e^{\frac{i m \vec{\eta}^{2}}{2 \hbar \varepsilon}} \vec{\eta}^{2} \eta_{x}^{2} \frac{\partial^{2} \Psi}{\partial x^{2}}+\frac{i}{2 \hbar} k \int \frac{d \vec{\eta}}{A^{3}} e^{\frac{i m \vec{\eta}^{2}}{2 \hbar \varepsilon}} \vec{\eta}^{2} \eta_{y}^{2} \frac{\partial^{2} \Psi}{\partial y^{2}} \\
& +\frac{i}{2 \hbar} k \int \frac{d \vec{\eta}}{A^{3}} e^{\frac{i m \vec{\eta}^{2}}{2 \hbar \varepsilon}} \vec{\eta}^{2} \eta_{z}^{2} \frac{\partial^{2} \Psi}{\partial z^{2}}
\end{aligned}
$$

In Eq. (40), the first term is

$$
\begin{aligned}
& \frac{i}{2 \hbar} k \int \frac{d \vec{\eta}}{A^{3}} e^{\frac{i m \vec{\eta}^{2}}{2 \hbar \varepsilon}} \vec{\eta}^{2} \eta_{x}^{2} \frac{\partial^{2} \Psi}{\partial x^{2}} \\
= & \frac{i}{2 \hbar} k \frac{\partial^{2} \Psi}{\partial x^{2}}\left[\int \frac{d \vec{\eta}}{A^{3}} e^{\frac{i m \vec{\eta}^{2}}{2 \hbar \varepsilon}}\left(\eta_{x}^{4}+\eta_{x}^{2} \eta_{y}^{2}+\eta_{x}^{2} \eta_{z}^{2}\right)\right] \\
= & \frac{i}{2 \hbar} k \frac{\partial^{2} \Psi}{\partial x^{2}}\left[\int \frac{d \eta_{x}}{A} \eta_{x}^{4} e^{\frac{i m \eta_{x}^{2}}{2 \hbar \varepsilon}} \int \frac{d \eta_{y}}{A} e^{\frac{i m \eta_{y}^{2}}{2 \hbar \varepsilon}} \int \frac{d \eta_{z}}{A} e^{\frac{i m \eta_{z}^{2}}{2 \hbar \varepsilon}}\right. \\
& +\int \frac{d \eta_{x}}{A} \eta_{x}^{2} e^{\frac{i m \eta_{x}^{2}}{2 \hbar \varepsilon}} \int \frac{d \eta_{y}}{A} \eta_{y}^{2} e^{\frac{i m \eta_{y}^{2}}{2 \hbar \varepsilon}} \int \frac{d \eta_{z}}{A} e^{\frac{i m \eta_{z}^{2}}{2 \hbar \varepsilon}} \\
& \left.+\int \frac{d \eta_{x}}{A} \eta_{x}^{2} e^{\frac{i m \eta_{x}^{2}}{2 \hbar \varepsilon}} \int \frac{d \eta_{y}}{A} e^{\frac{i m \eta_{y}^{2}}{2 \hbar \varepsilon}} \int \frac{d \eta_{z}}{A} \eta_{z}^{2} e^{\frac{i m \eta_{z}^{2}}{2 \hbar \varepsilon}}\right] .
\end{aligned}
$$

From Eq. (29), we can obtain the integral formula

$$
\int \frac{d \eta_{x}}{A} \eta_{x}^{4} e^{\frac{i m \eta_{x}^{2}}{2 \hbar \varepsilon}}=3\left(\frac{i \hbar \varepsilon}{m}\right)^{2} .
$$

From Eq. (26), (29) and (42), Eq. (41) becomes

$$
\begin{aligned}
& =\frac{i}{2 \hbar} k \frac{\partial^{2} \Psi}{\partial x^{2}}\left[3\left(\frac{i \hbar \varepsilon}{m}\right)^{2}+\left(\frac{i \hbar \varepsilon}{m}\right)^{2}+\left(\frac{i \hbar \varepsilon}{m}\right)^{2}\right] \\
& =5\left(\frac{i \hbar \varepsilon}{m}\right)^{2} \frac{i}{2 \hbar} k \frac{\partial^{2} \Psi}{\partial x^{2}} .
\end{aligned}
$$


The Eq. (43) is directly proportional to $\varepsilon^{2}$. Obviously, in Eq. (40), the second and third terms are also directly proportional to $\varepsilon^{2}$, and so the contribution of Eq. (40) can be neglected.

Substituting Eq. (31), (35) and (39) into (30), we can obtain

$$
\Psi(\vec{r}, t)+\varepsilon \frac{\partial \Psi(\vec{r}, t)}{\partial t}=\left(1-\frac{i \varepsilon}{\hbar} V(r, t)\right) \Psi(\vec{r}, t)+\frac{i \hbar}{2 m} \varepsilon \nabla^{2} \Psi-\frac{3 \varepsilon k}{m} \Psi(\vec{r}, t) .
$$

Equating the coefficient of powers of $\varepsilon$, we have

$$
\frac{\partial \Psi(\vec{r}, t)}{\partial t}=-\frac{i}{\hbar} V(r, t) \Psi(\vec{r}, t)+\frac{i \hbar}{2 m} \nabla^{2} \Psi-\frac{3 k}{m} \Psi(\vec{r}, t)
$$

multiplied by the coefficient of $i \hbar$, we have

$$
i \hbar \frac{\partial \Psi(\vec{r}, t)}{\partial t}=\left(-\frac{\hbar^{2}}{2 m} \nabla^{2}+V-i \hbar \frac{3 k}{m}\right) \Psi(\vec{r}, t)
$$

The Eq. (46) is a new quantum wave equation, which is suitable for the non-conservative force $\vec{F}=-k \vec{v}$. From the equation, we can study the non-conservative system.

\section{Conclusion}

We know Schrödinger's equation is quantum wave equation, which is only suitable for a conservative system. For a non-conservative system, we need a new quantum wave equation. In this paper, we apply the approach of path integral to obtain the general quantum wave equation, which is suitable for a non-conservative system. We think the new quantum wave equation should be used widely in the future. 


\section{References}

[1] R. P. Feynman, Rev. Mod. Phys., 20, (1948) 367.

[2] R. P. Feynman and A. R. Hibbs, Quantum mechanics and path integrals, McGrawHill Book Co.New York 1965.

[3] R. P. Feynman, Phys. Rev, 80, (1950) 440.

[4] P. Jordan, Z. Phys. 80 (1933) 285; P. Jordan and E. P. Wigner, Ann. Math. 35 (1934) 29.

[5] D. Finkelstein et al., Notes on Quaternion Quantum Mechanics (CERN, Report 597), in C. Hoker, ed. Logico-Algebraic-Approach to Quantum Mechanics $\amalg$ (Reidel, Dordrecht, 1979).

[6] D. Finkelstein et al., J. Math. Phys. 3 (1962) 207; ibid. 4 (1963) 788

[7] S. De Leo and K. Abdel-Khalek, Prog. Theor. Phys. 96 (1996) 823.

[8] D. Minic and C. -H. Tze, Phys. Lett. B 581 (2004) 111.

[9] F. Riewe, Phys. Rev. E 53, (1996) 1890.

[10] F. Riewe, Phys. Rev. E 55, (1997) 3581.

[11] H. Bateman, Phys. Rev. 38, (1931) 815.

[12] M. Razavy. Can. J. Phys. 56, (1978) 311.

[13] R. W. Hasse, J. Math. Phys. 16, (1975) 2005.

[14] H. Goldstein, Classical Mechanics (Addison-Wesley, Reading, MA, 1950) pp. 21-22.

[15] A. O. Caldeira and A. J. Leggett, Phys. Rev. Lett., 46, (1981) 211; Ann. Phys. N. Y. 149, (1983) 374; J. Ankerhold, H. Grabert, and G.-L. Ingold, Phys. Rev. E, 52, (1995) 4267.

[16] I. R. Senitzky, Phys. Rev. 119, (1960) 670 .

[17] K. W. H. Stevens, Proc. Phys. Soc. London 72, (1958)1027.

[18] G. W. Ford, M. Kac, and P. Mazur, J. Math. Phys. 6, (1965) 504; P. Ullersma, Physica (Utrecht) 32, (1966) 27.

[19] T. J. Krieger, Phys. Rev. 121, (1961) 1388.

[20] E. Celeghini, M. Rasetti, M. Tarlini, and G. Vitiello, Mod. Phys. Lett. B 3, (1989) 1213.

[21] H. B. Callen and T. A. Welton, Phys. Rev. 83, (1951) 34; L. H. Yu and C.-P. Sun, Phys. Rev. A 49, (1994) 592. 